\title{
Article
}

\section{The SADDEN DEATH Study: Results from a Pilot Study in Non-ICU COVID-19 Spanish Patients}

\author{
Carlos Nicolás Pérez-García ${ }^{1, *(1)}$, Daniel Enríquez-Vázquez ${ }^{1}$, Manuel Méndez-Bailón ${ }^{2}$, Carmen Olmos ${ }^{1}$, \\ Juan Carlos Gómez-Polo ${ }^{1}$, Rosario Iguarán ${ }^{1}$, Noemí Ramos-López ${ }^{2}$, José Luis García-Klepzig ${ }^{2}$ (1), \\ Marcos Ferrández-Escarabajal ${ }^{1}{ }^{10}$, Adrián Jerónimo ${ }^{1}$, Eduardo Martínez-Gómez ${ }^{1}{ }^{\mathbb{D}}$, Judit Font-Urgelles ${ }^{3}$, \\ Marcos Fragiel-Saavedra ${ }^{2}$, Pilar Paz-Arias ${ }^{2}$, Teresa Romero-Delgado ${ }^{1}$, Zaira Gómez-Álvarez ${ }^{1}$, \\ Julia Playán-Escribano 1일 , Esther Jaén ${ }^{2}$, Gianna Vargas ${ }^{4}$, Elizabeth González ${ }^{4}$, Eva Orviz ${ }^{2}$, Irene Burruezo ${ }^{2}$, \\ Alberto Calvo ${ }^{2}\left(\mathbb{0}\right.$, Ángel Nieto $^{2}$, Ángel Molino ${ }^{2}$, Noël Lorenzo-Villalba ${ }^{5}$, Emmanuel Andrès ${ }^{5}{ }^{(0)}$, Carlos Macaya ${ }^{1}$ \\ and Isidre Vilacosta ${ }^{1}$
}

Citation: Pérez-García, C.N.; Enríquez-Vázquez, D.; MéndezBailón, M.; Olmos, C.; Gómez-Polo, J.C.; Iguarán, R.; Ramos-López, N.; García-Klepzig, J.L.; Ferrández-

Escarabajal, M.; Jerónimo, A.; et al. The SADDEN DEATH Study: Results from a Pilot Study in Non-ICU COVID-19 Spanish Patients. J. Clin. Med. 2021, 10, 825. https://doi.org/ $10.3390 /$ jcm 10040825

Academic Editor: Sukhwinder Singh Sohal

Received: 17 January 2021

Accepted: 17 February 2021

Published: 18 February 2021

Publisher's Note: MDPI stays neutral with regard to jurisdictional claims in published maps and institutional affiliations.

Copyright: (c) 2021 by the authors. Licensee MDPI, Basel, Switzerland. This article is an open access article distributed under the terms and conditions of the Creative Commons Attribution (CC BY) license (https:// creativecommons.org/licenses/by/ $4.0 /)$.
1 Instituto Cardiovascular, Hospital Clínico San Carlos, 28040 Madrid, Spain; daniel.enriquez.vazquez@gmail.com (D.E.-V.); carmen.olmosblanco@gmail.com (C.O.); jc.gomezpolo@gmail.com (J.C.G.-P.); draiguaran@gmail.com (R.I.); marcos_ferres@hotmail.com (M.F.-E.); adrijeronimo@gmail.com (A.J.); emartinezg1@gmail.com (E.M.-G.); tererd9@gmail.com (T.R.-D.); zairagomez91@gmail.com (Z.G.-Á.); julia.playan@gmail.com (J.P.-E.); carlos.macaya@salud.madrid.org (C.M.); i.vilacosta@gmail.com (I.V.)

2 Servicio de Medicina Interna, Hospital Clínico San Carlos, 28040 Madrid, Spain; manuelmenba@hotmail.com (M.M.-B.); noemi.r192@gmail.com (N.R.-L.); josgar08@ucm.es (J.L.G.-K.); mfra2507@gmail.com (M.F.-S.); pilpazari23@gmail.com (P.P.-A.); estherjaen88@gmail.com (E.J.); evaorviz@gmail.com (E.O.); irene.burruezo@gmail.com (I.B.); ae.calvoelias@gmail.com (A.C.); anietosan@gmail.com (Á.N.); angelmanuel.molino@salud.madrid.org (Á.M.)

3 Servicio de Reumatología, Hospital Clínico San Carlos, 28040 Madrid, Spain; juditfu19@gmail.com

4 Servicio de Neumología, Hospital Clínico San Carlos, 28040 Madrid, Spain; gvargas@alumni.unav.es (G.V.); elisabethmanuely@gmail.com (E.G.)

5 Service de Médecine Interne, Diabète et Maladies Métaboliques, Hôpitaux Universitaires de Strasbourg, 67091 Strasbourg, France; noellorenzo@gmail.com (N.L.-V.); emmanuel.andres@chru-strasbourg.fr (E.A.)

* Correspondence: cnicolas.med@gmail.com; Tel.: +34-913302712; Fax: +34-913303290

Abstract: Introduction: The worldwide pandemic, coronavirus disease 2019 (COVID-19) is a novel infection with serious clinical manifestations, including death. Our aim is to describe the first non-ICU Spanish deceased series with COVID-19, comparing specifically between unexpected and expected deaths. Methods: In this single-centre study, all deceased inpatients with laboratory-confirmed COVID-19 who had died from March 4 to April 16, 2020 were consecutively included. Demographic, clinical, treatment, and laboratory data, were analyzed and compared between groups. Factors associated with unexpected death were identified by multivariable logistic regression methods. Results: In total, 324 deceased patients were included. Median age was 82 years (IQR 76-87); 55.9\% males. The most common cardiovascular risk factors were hypertension $(78.4 \%)$, hyperlipidemia $(57.7 \%)$, and diabetes $(34.3 \%)$. Other common comorbidities were chronic kidney disease $(40.1 \%)$, chronic pulmonary disease $(30.3 \%)$, active cancer $(13 \%)$, and immunosuppression $(13 \%)$. The Confusion, BUN, Respiratory Rate, Systolic BP and age $\geq 65$ (CURB-65) score at admission was $>2$ in $40.7 \%$ of patients. During hospitalization, $77.8 \%$ of patients received antivirals, $43.3 \%$ systemic corticosteroids, and $22.2 \%$ full anticoagulation. The rate of bacterial co-infection was 5.5\%, and $105(32.4 \%)$ patients had an increased level of troponin I. The median time from initiation of therapy to death was 5 days (IQR 3.0-8.0). In 45 patients (13.9\%), the death was exclusively attributed to COVID-19, and in 254 patients (78.4\%), both COVID-19 and the clinical status before admission contributed to death. Progressive respiratory failure was the most frequent cause of death (92.0\%). Twenty-five patients $(7.7 \%)$ had an unexpected death. Factors independently associated with unexpected death were male sex, chronic kidney disease, insulin-treated diabetes, and functional independence. Conclusions: This case series provides in-depth characterization of hospitalized non-ICU COVID-19 patients who died in Madrid. Male sex, insulin-treated diabetes, chronic kidney disease, and independency for activities of daily living are predictors of unexpected death. 


\section{Introduction}

Based on data reported by the John Hopkins University Coronavirus Resource Center, severe acute respiratory syndrome coronavirus 2 (SARS-CoV-2) has caused more than two million deaths in 221 countries [1]. Spain, with more than 2,900,000 cases and 60,000 deaths as of February 2021, has one of the highest burdens of COVID-19 worldwide. Madrid has the highest rate of death per capita in Spain [2].

At the hospital, with the massive admission of patients, we are struggling with limited ICU capacity and resources. We prioritize patients who are more likely to survive over those with much lower chances of survival. Such prioritization inevitably leads to some patients dying from progressive respiratory failure in the hospital wards. While we were taking care of these patients, we realized that some died suddenly and unexpectedly, and that hypoxemia did not sufficiently explain the ultimate reason for death. This fact, together with the social and emotional impact of isolation during the first wave of COVID19 pandemic, explains the name of our study. SADDEN is the result of the combination of "Sudden" and "Sad", as most patients died alone and away from their families.

COVID-19-related deaths are not clearly defined in the available international reports. Most articles are related to critically ill ICU patients [3,4]. Thus, there is not much information on how patients die on general medical wards. Specifically, nothing has been published about sudden death and COVID-19. Our hypothesis was that some of the unexpected deaths could be cardiovascular in origin.

The purpose of this study was to describe the demographics, baseline comorbidities, clinical presentation, laboratory data, and other complementary test results of the first deceased series of hospitalized patients from an academic hospital in Spain. Specifically, we aimed to compare patients with unexpected and expected deaths.

\section{Methods}

\subsection{Study Design and Participants}

A retrospective study was conducted at Clínico San Carlos Hospital in Madrid. Patients with confirmed COVID-19 by positive reverse transcriptase-polymerase chain reaction (RT-PCR) of a nasopharyngeal sample who required hospital admission between 4 March 2020, and 16 April 2020 were included.

The flow of patients was: (1) from the Emergency Department (ED); most patients were discharged or admitted to hospital, and a minority died there;(2) among hospitalized patients, we distinguished four subgroups: patients with severe disease admitted to ICU, patients with severe disease, not admitted to ICU because of lack of resources, patients with severe disease and poor prognosis, who were not candidates for intensive care, and patients with disease not severe enough to warrant ICU; (3) Our study cohort was formed by those patients from the last three subgroups who died in our general medical wards. Due to their different clinical situation and treatment, patients who died in the ICU and in ED were excluded.

This study was approved by the local ethical committee (protocol code 20/342E_COVID, 17 April 2020), and the study protocol was carried out in accordance with The Code of Ethics of the World Medical Association (Declaration of Helsinki).

\subsection{Data Collection}

A comprehensive multidisciplinary investigation of the clinical course, complementary test results and premortem data was performed. Data were retrospectively collected from electronic medical records and patient files; daily reports from nurses and doctors were carefully reviewed. Data included patient demographics, comorbidities, home medications, clinical status on admission, initial laboratory tests, initial electrocardiogram (12-lead 
ECG), inpatient medications, evolutionary ECG changes, transthoracic echocardiography, computed tomography angiography (CTA), detailed clinical evolution during the hospital course, emphasizing the last $24 \mathrm{~h}$ before death, length of stay, and the mode of death. For purposes of comparison, we distinguished two groups: Group I (patients with unexpected death), and Group II (expected death). The study was divided into three equal periods of a fortnight, as treatment and ICU resources varied along the study period. All data were recorded on a multipurpose database exclusively created for this study.

\subsection{Definitions}

Patients were considered to have confirmed infection if the initial RT-PCR test result was positive, or if it was negative but repeat testing was positive. Initial laboratory testing was defined as the first test results available, typically within $24 \mathrm{~h}$ of admission. All patients had chest X-ray and ECG at admission and, in most cases, during hospitalization. For those laboratory results, electrocardiographic traces, and other studies for which not all patients had values, percentages of total patients with completed tests are shown.

The CURB-65 score was used for the assessment of the severity of pneumonia at admission [5]. Cognitive impairment was assessed according to the Mini-Mental State Examination administered before admission [6]. Sepsis and septic shock were defined according to the 2016 Third International Consensus Definition for Sepsis and Septic Shock. Secondary infection was diagnosed when the patient had a positive culture from lower respiratory tract specimens or blood samples [7]. Resistant hypertension and chronic kidney disease (CKD) were defined according to the European Society of Cardiology guidelines [8]. Acute respiratory distress syndrome was diagnosed according to the Berlin definition [9]. Difficulty with activities of daily living (ADL) were divided into three categories (no, partial and total).

All investigators participating in this study were also deeply involved in the care of these patients. The actual role of COVID-19 in the patient's death was classified into three groups according to the infection severity, comorbidity burden and life expectancy: Group A (no cancer, absence of comorbidities or decompensated underlying disease, and life expectancy $\geq 1$ y); Group B (active cancer, decompensated comorbidities and/or life expectancy $<1 \mathrm{y}$ ), and Group $C$ (the underlying condition was the cause of death and the infection behaved as an epiphenomenon).

\subsection{Definition of Unexpected Death}

In this study, unexpected death was defined as a sudden, non-traumatic death caused by cardiac or unknown cause [10]. Although the term "sudden cardiac death" could be a synonym, we preferred to employ the term "unexpected death", due to the particular scenario (with severe hypoxemia) of many of these patients, and the limitations on performing autopsies, especially during the first wave of the pandemic.

The death of these patients (Group I) contrasts with Group II, patients who die presenting progressive respiratory failure, often accompanied by medical sedation; ischemic or hemorrhagic stroke; sepsis; progressive heart failure; coagulopathy or multiorgan failure.

In order to be considered as unexpected death, the criteria used were as follows: in unwitnessed cases, the person had to have been seen as clinically stable $<2 \mathrm{~h}$ before being found dead, and in witnessed cases, the patient had to have an acute change in his hemodynamic status and/or any cardiovascular symptom (chest pain, syncope, sudden breathlessness, etc.) with the time to death being $<1 \mathrm{~h}$. In doubtful cases, a multidisciplinary team made up of three cardiologists (CP, DE, IV), one internist (MM), and the researcher in charge of collecting patients' data, made the final decision.

\subsection{Statistical Analysis}

Categorical variables are expressed as a frequency and a percentage and compared with the chi-square test and Fisher's exact test as appropriate. Continuous variables are expressed as median and interquartile range (IQR) or mean and standard deviation 
(SD). Assessment of the normality and equality of variances for continuous data was performed using the Shapiro-Wilk test. Thereafter, continuous variables were compared using Student's $t$-test, the Mann-Whitney U test or Wilcoxon test. To identify the variables associated with unexpected death, univariable and multivariable logistic regression models were used. Variables that were statistically significant in the univariable analysis $(p<0.05)$, or considered clinically relevant, were integrated in a multivariable regression model. The adjusted odds ratios (ORs) with 95\% confidence intervals (CIs) for each variable were calculated.

All tests were two-sided, and differences were considered statistically significant at $p$ values $<0.05$. Statistical analysis was performed with Stata v14.1 (StataCorp, College Station, TX, USA).

\section{Results}

\subsection{Patient Characteristics and Pre-Hospital Condition}

Out of 1946 adult patients who were hospitalized between March 3 and April 16 2020 with COVID-19, we obtained data from 387 patients who died in hospital. After excluding 33 patients who died in the ICU, 16 in the ED, and 14 inpatients without available key information or confirmed infection, we included 324 deceased patients in the final analysis. The demographics and patients' clinical characteristics are shown in Table 1. The median age was 82.8 years (IQR 76-87), ranging from 46 years to 105 years; $55.9 \%$ were male. Importantly, one hundred and twenty-six patients $(39.1 \%)$ were partially or totally dependent in ADL, and 49 (15\%) were institutionalized. Twenty-two cases (6.8\%) were nosocomial.

The global burden of cardiovascular diseases and risk factors was significant $(65.4 \%$ had $\geq 2$ main cardiovascular risk factors), with hypertension being the most common cardiovascular risk factor, followed by type-2 diabetes mellitus (T2DM), in 34.3\%. Ninety-eight patients $(30.3 \%)$ had chronic pulmonary disease (CPD) and $22(6.8 \%)$ of them received home supplemental oxygen. One hundred and thirty patients $(40.1 \%)$ had CKD, with nine in hemodialysis. Eighty-one patients (25\%) were on anticoagulants and $176(55 \%)$ were taking an angiotensin-converting enzyme inhibitor (ACE-i) or an angiotensin II receptor blocker (ARB) at home. Additional home medication can be found in the Supplementary Material.

\subsection{Admission and Clinical Evolution}

The median time from symptom onset to admission was 5 days (IQR 3-8). Patient's characteristics at admission and clinical evolution are shown in Table 2. One hundred and thirty-two patients $(40.7 \%)$ had a CURB-65 score $\geq 3$ at admission. The most common symptoms on admission were fever, dyspnea, cough, and fatigue. Two hundred and twelve patients (66\%) had bilateral pneumonia; other radiological findings are shown in the Supplementary Material. Thirty-eight patients $(18.5 \%)$ were in atrial fibrillation. Seventeen patients $(5.5 \%)$ had bacterial co-infection. Most patients had lymphocytopenia, and elevated levels of D-dimer, serum ferritin and C-reactive protein (Table 2). One hundred and five (32.4\%) patients had an increased level of troponin I $(>0.05 \mathrm{ng} / \mathrm{mL})$ during hospitalization. Two hundred and fifty-two patients (77.8\%) received antivirals, and $142(43.8 \%)$ received corticosteroids. Both the use of corticosteroids and prophylactic anticoagulation were greater in the last fortnight than in the first two $(50.7 \%$ vs. $7.0 \%$ and $42.3 \%, p<0.001$, and $62.0 \%$ vs. $26.3 \%$ and $46.7 \%, p 0.003$, respectively). Corticosteroids were more frequently used when ferritin value was $\geq 1500 \mathrm{ng} / \mathrm{mL}$ (58.7\% vs. $43.8 \%$; $p$ 0.04). Prophylactic anticoagulation was less frequently prescribed in patients with CKD (46.8\% vs. $66.3 \%, p$ 0.02). Of the patients taking ACE-i or ARB at home, 68 continued taking these medications during their hospitalization. Fourteen patients had a QTc interval $>450 \mathrm{~ms}$, and only two had a QTc >500 ms; ventricular arrhythmias were not documented in these patients. 
Table 1. Baseline characteristics of study population.

\begin{tabular}{|c|c|}
\hline Baseline Characteristics & $\begin{array}{l}\text { Total of Deaths } \\
\quad(n=324)\end{array}$ \\
\hline \multicolumn{2}{|l|}{ Demographic characteristics } \\
\hline $\begin{array}{c}\text { Age (median (IQR))-y. } \\
\text { Age (years) }\end{array}$ & $82.8(76.5-87.3)$ \\
\hline Male gender-no. (\%) & $181(55.9)$ \\
\hline \multicolumn{2}{|l|}{ Frailty indicators } \\
\hline \multicolumn{2}{|l|}{ Functional dependency-no. (\%) $n=322$} \\
\hline Independent & $196(60.9)$ \\
\hline Partially dependent & $87(27)$ \\
\hline Totally dependent & $39(12.1)$ \\
\hline Institutionalization-no. (\%) & $49(15.1)$ \\
\hline Cognitive impairment-no. (\%) & $74(22.8)$ \\
\hline \multicolumn{2}{|l|}{ Cardiovascular risk factors } \\
\hline Hypertension-no. (\%) & $254(78.4)$ \\
\hline Resistant hypertension-no. (\%) & $38(11.7)$ \\
\hline T2DM-no. (\%) & $111(34.3)$ \\
\hline T2DM-OAD & $90(27.8)$ \\
\hline T2DM-insulin & $47(14.5)$ \\
\hline Hyperlipidemia-no. (\%) & 187 (57.7) \\
\hline Obesity-no. (\%) & $60(18.5)$ \\
\hline Current smoking-no. $(\%)$ & $15(4.6)$ \\
\hline Former smoking-no. (\%) & $95(30.9)$ \\
\hline$<2$ CVRF-no. $(\%)$ & $112(34.6)$ \\
\hline$\geq 2$ CVRF-no. $(\%)$ & $212(65.4)$ \\
\hline \multicolumn{2}{|l|}{ Cardiovascular history } \\
\hline Ischaemic heart disease-no. (\%) & $40(12.3)$ \\
\hline Prior MI-no. $(\%)$ & $28(8.6)$ \\
\hline Prior stroke/TIA—no. $(\%)$ & $37(11.4)$ \\
\hline Atrial fibrillation-no. (\%) & $62(19.1)$ \\
\hline Pacemaker-no. $(\%)$ & $19(5.9)$ \\
\hline $\mathrm{ICD} \pm \mathrm{CRT}$-no. $(\%)$ & $2(0.6)$ \\
\hline LVEF $<35 \% n=198$ & $8(4.0)$ \\
\hline \multicolumn{2}{|l|}{ Other comorbidities } \\
\hline Chronic pulmonary disease-no. $(\%)$ & $98(30.3)$ \\
\hline COPD & $49(15.1)$ \\
\hline Asthma & $14(4.3)$ \\
\hline OSAS & $16(4.9)$ \\
\hline Restrictive disorder & $15(4.6)$ \\
\hline
\end{tabular}


Table 1. Cont.

\begin{tabular}{cc}
\hline Baseline Characteristics & $\begin{array}{c}\text { Total of Deaths } \\
(\boldsymbol{n}=\mathbf{3 2 4})\end{array}$ \\
\hline Chronic kidney disease-no. (\%) & $130(40.1)$ \\
\hline Chronic liver disease-no. (\%) & $17(5.2)$ \\
\hline Rheumatological disease—no. (\%) & $31(9.6)$ \\
\hline Hypothyroidism-no. (\%) & $38(11.7)$ \\
\hline Active cancer-no. (\%) & $42(13.0)$ \\
\hline $\begin{array}{l}\text { Immulues are no (\%) or median (interquartile range) as indicated. }{ }^{*} \text { Combination of immunosuppressive therapies } \\
\text { (corticosteroids and other immunosuppressive drugs), transplanted patients and HIV+. COPD: chronic obstructive } \\
\text { pulmonary disease, CRT: cardiac resynchronization therapy, CVRF: cardiovascular risk factor, HIV: human } \\
\text { immunodeficiency virus, ICD: implantable cardioverter defibrillator; MI: myocardial infarction, no: number, } \\
\text { OSAS: obstructive sleep apnea syndrome, T2DM: type-2 diabetes mellitus. }\end{array}$
\end{tabular}

Table 2. Patient characteristics during admission.

\begin{tabular}{|c|c|}
\hline Patient Characteristics during Hospitalization & $\begin{array}{l}\text { Total of Deaths } \\
\quad(n=324)\end{array}$ \\
\hline \multicolumn{2}{|l|}{ Clinical presentation } \\
\hline Fever $\geq 38^{\circ} \mathrm{C}-$ no. $(\%)$ & $214(66.3)$ \\
\hline Cough-no. (\%) & $191(59.1)$ \\
\hline Dyspnea-no. (\%) & $204(63.0)$ \\
\hline Asthenia-no. (\%) & $139(43.0)$ \\
\hline Anosmia-no. (\%) & $3(0.9)$ \\
\hline Ageusia-no. (\%) & $6(1.9)$ \\
\hline Gastrointestinal symptoms-no. (\%) & $91(28.1)$ \\
\hline Syncope-no. (\%) & $12(3.7)$ \\
\hline Myalgia-no. (\%) & $42(13.0)$ \\
\hline Confusion-no. (\%) & $91(28.4)$ \\
\hline Time from symptoms to admission (median (IQR))-days. & $5(3-8)$ \\
\hline \multicolumn{2}{|l|}{ Clinical situation at admission } \\
\hline CURB-65 > 2-no. (\%) & $132(40.7)$ \\
\hline $\mathrm{pH}$ (venous sample) (mean $\pm \mathrm{SD}$ ) & $7.4 \pm 0.1$ \\
\hline pCO2 (venous sample, $\mathrm{mmHg}$ ) (mean $\pm \mathrm{SD})$ & $40.7 \pm 9.9$ \\
\hline Capillary oxygen saturation (\%) (mean \pm SD) & $87.2 \pm 9.1$ \\
\hline Systolic blood pressure $(\mathrm{mmHg})($ mean $\pm \mathrm{SD})$ & $127.8 \pm 23.9$ \\
\hline Diastolic blood pressure $(\mathrm{mmHg})($ mean $\pm \mathrm{SD})$ & $70.2 \pm 13.9$ \\
\hline Tachypnea-no. (\%) & $150(47.9)$ \\
\hline $\begin{array}{l}\text { Abnormal pulmonary auscultation (rales, hypophonesis and } \\
\text { rhonchus)-no. (\%) }\end{array}$ & $296(91.4)$ \\
\hline $\begin{array}{l}\text { Time from symptoms to initiation of therapy (median } \\
\text { (IQR))-days. }\end{array}$ & $5(2-7)$ \\
\hline \multicolumn{2}{|l|}{ Clinical evolution ( $24 \mathrm{~h}$ before death) } \\
\hline Acute respiratory and radiological worsening-no. (\%) & $299(92.9)$ \\
\hline Pronation in bed (awake)—no. (\%) & $58(18.0)$ \\
\hline Hemodynamic instability-no. (\%) & $115(36.5)$ \\
\hline New onset arrhythmias-no. (\%) & $8(2.6)$ \\
\hline Neurological deterioration-no. (\%) & $100(32.8)$ \\
\hline
\end{tabular}


Table 2. Cont.

\begin{tabular}{|c|c|c|c|c|}
\hline Patient Characteristics during Hospitalization & \multicolumn{4}{|c|}{$\begin{array}{l}\text { Total of Deaths } \\
\quad(n=324)\end{array}$} \\
\hline Acute renal injury-no. (\%) & \multicolumn{4}{|c|}{$94(30.2)$} \\
\hline Chest pain-no. (\%) & \multicolumn{4}{|c|}{$7(2.2)$} \\
\hline Syncope-no. (\%) & \multicolumn{4}{|c|}{$4(1.2)$} \\
\hline Length of hospitalization (median (IQR))—days. & \multicolumn{4}{|c|}{$5(2-9)$} \\
\hline Time from initiation of therapy to death (median (IQR))—days. & \multicolumn{4}{|c|}{$5(3-8)$} \\
\hline \multicolumn{5}{|l|}{ Laboratory findings } \\
\hline Hemoglobin at admission (mean $\pm \mathrm{SD}$ )—g/dL & \multicolumn{4}{|c|}{$13.0 \pm 2.1$} \\
\hline Leucocytes at admission (mean $\pm \mathrm{SD}$ )—cells $/ \mathrm{mm}^{3}$ & \multicolumn{4}{|c|}{$8757.8 \pm 7427.4$} \\
\hline Platelets at admission (mean $\pm \mathrm{SD}$ )—cells $/ \mathrm{mm}^{3}$ & \multicolumn{4}{|c|}{$185,467 \pm 90,082$} \\
\hline $\mathrm{CRP}$ at admission (mean $\pm \mathrm{SD})-\mathrm{mg} / \mathrm{dL}$ & \multicolumn{4}{|c|}{$13.9 \pm 10.4$} \\
\hline Procalcitonin at admission [median (IQR)]—ng/mL & \multicolumn{4}{|c|}{$0.3(0.1-0.7)$} \\
\hline Creatinine at admission (mean $\pm \mathrm{SD}$ )—mg/dL & \multicolumn{4}{|c|}{$1.6 \pm 1.6$} \\
\hline Urea at admission (mean $\pm \mathrm{SD}$ ) $-\mathrm{mg} / \mathrm{dL}$ & \multicolumn{4}{|c|}{$85.2 \pm 87.0$} \\
\hline Maximum lymphocytopenia *—(median $(\mathrm{IQR}))$ —cells $/ \mathrm{mm}^{3}$ & \multicolumn{4}{|c|}{$500(300-700)$} \\
\hline Peak Troponin I + (mean $\pm \mathrm{SD})-\mathrm{ng} / \mathrm{mL}$ & \multicolumn{4}{|c|}{$2.4 \pm 26.1$} \\
\hline Peak D-dimer (mean \pm SD)-ng $/ \mathrm{mL}$ & \multicolumn{4}{|c|}{$10,808 \pm 25646$} \\
\hline Peak Ferritin (mean $\pm \mathrm{SD})-\mathrm{ng} / \mathrm{mL}$ & \multicolumn{4}{|c|}{$1385 \pm 1673$} \\
\hline Peak Triglycerides (peak) (mean \pm SD)-mg/dL & \multicolumn{4}{|c|}{$179.9 \pm 138.0$} \\
\hline IL-6 (median (IQR))—ng/mL & \multicolumn{4}{|c|}{$86.3(51.5-273)$} \\
\hline INR ( $24 \mathrm{~h}$ before death) (mean $\pm \mathrm{SD}$ ) & \multicolumn{4}{|c|}{$1.7 \pm 2.1$} \\
\hline APTT ( $24 \mathrm{~h}$ before death) (mean $\pm \mathrm{SD})-\mathrm{s}$ & \multicolumn{4}{|c|}{$30.7 \pm 9.4$} \\
\hline \multicolumn{5}{|l|}{ ECG findings } \\
\hline Sinus rhythm-no. (\%) & \multicolumn{4}{|c|}{$162(79.4)$} \\
\hline QT interval corrected $\ddagger($ mean \pm SD $)$ 一 ms. & \multicolumn{4}{|c|}{$403.0 \pm 38.6$} \\
\hline PR interval (mean \pm SD)—ms. & & 191.4 & 35.7 & \\
\hline Treatment during hospitalization & & & & \\
\hline Hydroxychloroquine-no. (\%) & & $215(6$ & & \\
\hline Lopinavir/ritonavir-no. (\%) & & $111(3$ & & \\
\hline Darunavir/cobicistat-no. (\%) & & $23(7$ & & \\
\hline Azithromycin-no. (\%) & & $93(2$ & & \\
\hline Corticosteroids-no. (\%) & & $142(4$ & & \\
\hline Tocilizumab-no. (\%) & & $15(4$ & & \\
\hline Therapeutic anticoagulation (AVK, heparin and DOAC)-no. (\%) & & $72(2$ & & \\
\hline Therapeutic anticoagulation per fortnight-no. (\%) & $\begin{array}{l}\text { 1st } 4 \\
(21.1) \\
\end{array}$ & $\begin{array}{c}\text { 2nd } 40 \\
(21.7)\end{array}$ & $\begin{array}{c}3 \text { rd 28 } \\
(23.1) \\
\end{array}$ & $p 0.95$ \\
\hline Prophylactic anticoagulation per fortnight-no. (\%) & $\begin{array}{l}\text { 1st } 5 \\
(26.3) \\
\end{array}$ & $\begin{array}{c}\text { 2nd } 86 \\
(46.7)\end{array}$ & $\begin{array}{c}3 \text { rd 75 } \\
(62.0)\end{array}$ & $\begin{array}{c}p \\
0.003 \\
\end{array}$ \\
\hline COVID-19 death characteristics & & & & \\
\hline Death mainly from COVID-19 (Group A)—no. (\%) & & $45(1$ & & \\
\hline Death from COVID-19 with comorbidity (Group B)—no. (\%) & & $254(7$ & & \\
\hline Death with COVID-19 (Group C) §—no. (\%) & & $25(7$ & & \\
\hline Progressive respiratory failure to death-no. (\%) & & $298(C$ & & \\
\hline Unexpected death—no. (\%) & & $25(7$ & & \\
\hline Death at night (22-8 h)-no. (\%) & & $116(4$ & & \\
\hline
\end{tabular}


Table 2. Cont.

\begin{tabular}{|c|c|c|c|c|}
\hline Patient Characteristics during Hospitalization & \multicolumn{4}{|c|}{$\begin{array}{l}\text { Total of Deaths } \\
\quad(n=324)\end{array}$} \\
\hline \multicolumn{5}{|l|}{ Reasons for non-admission to the ICU } \\
\hline Age-no. (\%) & \multicolumn{4}{|c|}{$240(74.5)$} \\
\hline High comorbidity一no. (\%) & \multicolumn{4}{|c|}{$240(74.5)$} \\
\hline Cancer-no. $(\%)$ & \multicolumn{4}{|c|}{$52(16.0)$} \\
\hline Frailty-no. (\%) & \multicolumn{4}{|c|}{$185(57.1)$} \\
\hline Cognitive impairment-no. $(\%)$ & \multicolumn{4}{|c|}{$74(22.8)$} \\
\hline Non-available ICU bed-no. (\%) & \multicolumn{4}{|c|}{$59(18.2)$} \\
\hline Non-available ICU bed per fortnight-no. (\%) N = 59 & $\begin{array}{l}\text { 1st } 6 \\
(10.1)\end{array}$ & $\begin{array}{l}\text { 2nd } 32 \\
(54.2)\end{array}$ & $\begin{array}{l}\text { 3rd } 21 \\
(35.6)\end{array}$ & $p 0.33$ \\
\hline ICU team assessment-no. (\%) & \multicolumn{4}{|c|}{$48(14.8)$} \\
\hline \multicolumn{5}{|c|}{$\begin{array}{l}\text { Values are } \mathrm{n}(\%) \text {, mean ( } \pm \text { standard deviation) or median (interquartile range) as indicated. }{ }^{*} \text { Five patients were } \\
\text { excluded ( } 3 \text { with chronic lymphocytic leukemia and } 2 \text { with lymphomas). }+ \text { A value }>0.05 \mathrm{ng} / \mathrm{mL} \text { was considered } \\
\text { abnormal. } \ddagger \text { Bazett formula. \& Short life-expectancy before the infection. APTT: activated partial thromboplastin } \\
\text { time, AVK: antivitamin K, CRP: C-reactive protein, DOAC: direct oral anticoagulant, ICU: Intensive Care Unit } \\
\text { INR: international normalized ratio, no: number, pCO2: partial pressure of carbon dioxide. }\end{array}$} \\
\hline
\end{tabular}

The overall length of hospital stay was 5 days (IQR 2-9). Deaths were more frequent in the second fortnight of the study $(n=184,56.8 \%)$ (Figure 1$)$. The most frequent cause of death was progressive respiratory failure $(n=298,92.0 \%)$. Unexpected death occurred in 25 patients $(7.7 \%)$. When we analyzed the actual contribution of the virus to the patient's death, we found that, in 45 patients $(13.9 \%)$ (Group A), the death was exclusively attributed to COVID-19, and in 254 patients (78.4\%) (Group B), both COVID-19 and the patient's clinical situation before admission contributed to death (Table 2). As expected, median age was lower in Group A than in Group B (74.9 vs. 84.2 y, $p<0.001)$. Cognitive impairment and active cancer were more common in Group B $(2.2 \%$ vs. $26.1 \%, p<0.001$, and $0 \%$ vs. $54.7 \%$, $p 0.03$, respectively). All patients from Group A were independent for ADL, whereas in Group B only $56.8 \%$ were functionally independent. There were 25 patients with COVID-19 (Group C) whose death was independent of SARS-CoV-2 infection (cardiogenic shock, multiorgan failure, aspiration pneumonia, medullary aplasia, hemorrhagic stroke, etc.).

The reason the patient was not admitted to the ICU is shown in Table 2. Increased age, high comorbidity (e.g., cirrhosis, hemodialysis, severe COPD), frailty, and cognitive impairment were the most frequent reasons. Most patients from Group B were not candidates for mechanical ventilation. Any patient over 70 years old was a borderline candidate for intensive care treatment. However, the age threshold for being an ICU candidate varied throughout the study period depending on resources, and during the period of the study, $18 \%$ of our patients were pronated on the general ward.

Among patients from Group A, the main reason for not being admitted to the ICU was the lack of ICU beds in $67.4 \%$ of cases.

\subsection{Unexpected Death}

Patients with unexpected death had a median age of 82.1 years (72.2-87.3), and 21 were male $(84 \%)$. The comparison of clinical characteristics, treatment and complications between patient groups are shown in Table 3. The proportion of unexpected deaths in relation to those with expected death was higher in the first study period $(26.3 \%$ vs. $6.0 \%$ vs. $7.4 \%, p 0.01)$. Patients with unexpected death were more frequently independent in ADL than those with expected death, whereas cognitive impairment and confusion were more common in the latter. Three patients collapsed in the bathroom and were discovered by the nurse later. 


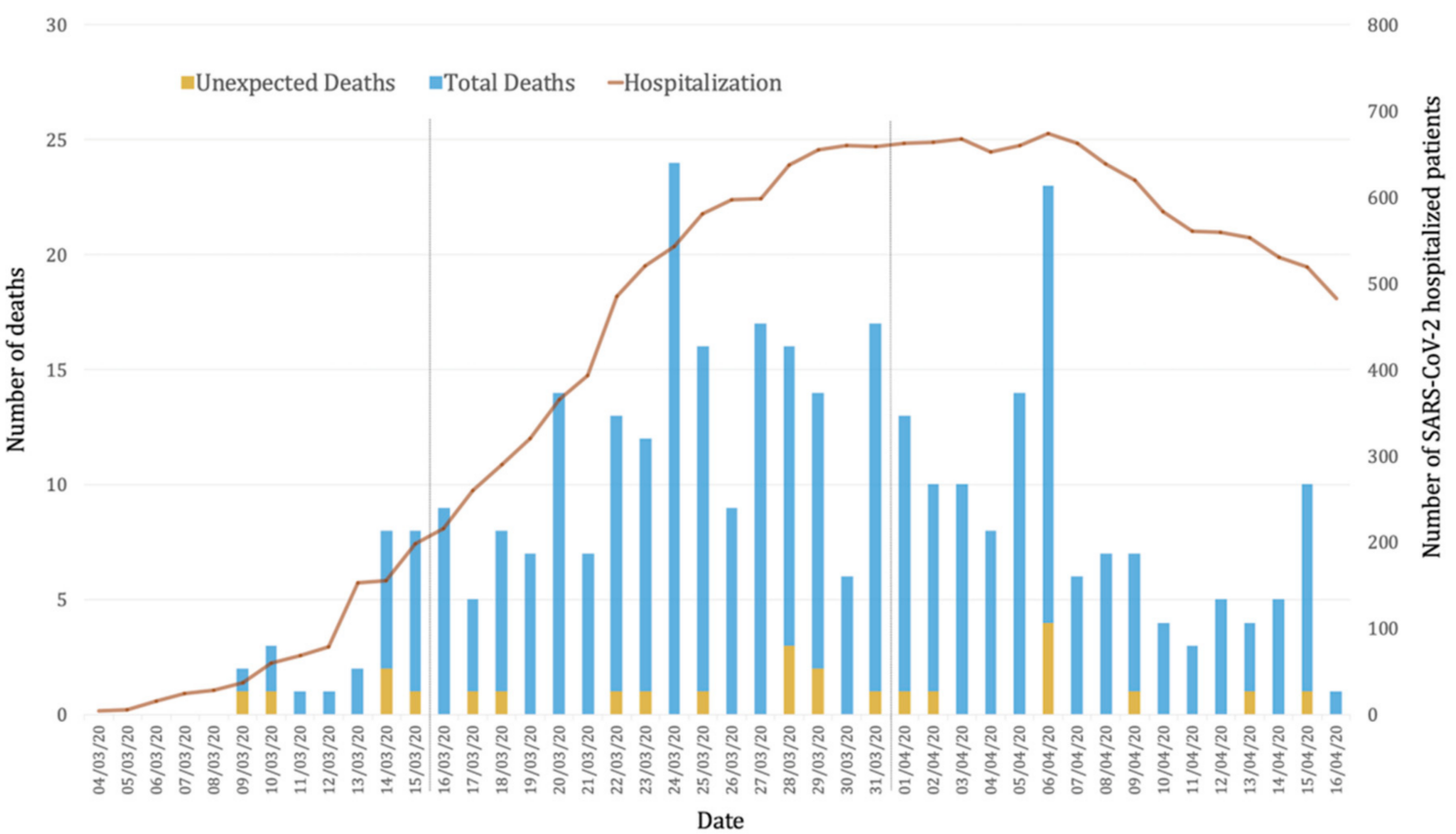

Figure 1. In-hospital mortality in patients with SARS-CoV-2 infection during the study period. The daily number of unexpected deaths (yellow), non-unexpected deaths (blue) and number of COVID-19 hospitalized patients are represented and divided by fortnight.

Table 3. Univariable analysis between patients with unexpected death and non-unexpected death.

\begin{tabular}{|c|c|c|c|}
\hline Univariable Analysis & $\begin{array}{l}\text { Unexpected } \\
\text { Deaths } \\
(n=25)\end{array}$ & $\begin{array}{c}\text { Non-Unexpected } \\
\text { Deaths } \\
(n=299)\end{array}$ & $p$-Value \\
\hline \multicolumn{4}{|l|}{ Demographic characteristics } \\
\hline Age (median, (IQR))—y. & $\begin{array}{c}82.1 \\
(72.2-87.3)\end{array}$ & $82.8(76.8-87.5)$ & 1.00 \\
\hline Male gender-no. $(\%)$ & $21(84.0)$ & $160(53.5)$ & 0.003 \\
\hline \multicolumn{4}{|l|}{ Frailty indicators } \\
\hline Functional independence-no. (\%) & $20(80.0)$ & $176(59.3)$ & 0.04 \\
\hline Cognitive impairment-no. (\%) & $2(8.0)$ & $72(24.2)$ & 0.08 \\
\hline \multicolumn{4}{|l|}{ Cardiovascular risk factors } \\
\hline Hypertension-no. (\%) & $21(84.0)$ & $233(77.9)$ & 0.62 \\
\hline Diabetes mellitus 2-no. (\%) & $14(56.0)$ & $97(32.4)$ & 0.02 \\
\hline T2DM-OAD & $7(28.0)$ & $83(27.8)$ & 0.98 \\
\hline T2DM-insulin requiring & $8(32.0)$ & $39(13.0)$ & 0.01 \\
\hline Former smoking-no. (\%) & $12(48.0)$ & $83(29.3)$ & 0.05 \\
\hline 2 CVRF-no. (\%) & $21(84.0)$ & $191(63.9)$ & 0.049 \\
\hline \multicolumn{4}{|l|}{ Cardiovascular events } \\
\hline Prior MI-no. $(\%)$ & $1(4.0)$ & $27(9.1)$ & 0.71 \\
\hline Prior stroke/TIA-no. $(\%)$ & $2(8.0)$ & $35(11.7)$ & 0.75 \\
\hline
\end{tabular}


Table 3. Cont.

\begin{tabular}{|c|c|c|c|}
\hline Univariable Analysis & $\begin{array}{l}\text { Unexpected } \\
\text { Deaths } \\
(n=25)\end{array}$ & $\begin{array}{l}\text { Non-Unexpected } \\
\text { Deaths } \\
(n=299)\end{array}$ & $p$-Value \\
\hline Heart failure ${ }^{*}-$ no. $(\%)$ & $5(21.7)$ & $55(19.3)$ & 0.79 \\
\hline Venous thromboembolic disease (PE or DVT) & $1(4.0)$ & $23(7.7)$ & 1.00 \\
\hline \multicolumn{4}{|l|}{ Other comorbidities } \\
\hline Chronic pulmonary disease-no. (\%) & $12(48.0)$ & $86(28.8)$ & 0.04 \\
\hline Chronic kidney disease-no. (\%) & $16(64.0)$ & $114(38.13)$ & 0.01 \\
\hline Chronic liver disease-no. (\%) & $2(8.0)$ & $15(5.0)$ & 0.63 \\
\hline Active cancer-no. (\%) & $4(16.0)$ & $38(12.7)$ & 0.55 \\
\hline Immunosuppression $†$-no. $(\%)$ & $7(28.0)$ & $48(16.1)$ & 0.13 \\
\hline \multicolumn{4}{|l|}{ Baseline medications } \\
\hline RAAS inhibitors $\ddagger$ no. (\%) & $13(52.0)$ & $161(53.9)$ & 0.86 \\
\hline Antibiotics with effects on QT-no. (\%) & $3(12.0)$ & $35(11.7)$ & 1.00 \\
\hline Antipsychotic drugs-no. $(\%)$ & $0(0.0)$ & $34(11.4)$ & 0.09 \\
\hline \multicolumn{4}{|l|}{ Clinical presentation } \\
\hline Fever-no. (\%) & $16(64.0)$ & $198(66.4)$ & 0.80 \\
\hline Oxygen saturation (mean, (SD)) & $87.1( \pm 9.2)$ & $87.2( \pm 9.1)$ & 0.97 \\
\hline Tachypnea-no. (\%) & $12(50.0)$ & $138(47.8)$ & 0.83 \\
\hline Confusion-no. (\%) & $5(20.0)$ & $86(29.2)$ & 0.05 \\
\hline Abnormal pulmonary auscultation-no. (\%) & $20(80.0)$ & $276(92.3)$ & 0.05 \\
\hline CURB-65 > 2-no. (\%) & $10(40.0)$ & $122(40.8)$ & 0.94 \\
\hline $\begin{array}{l}\text { Time from symptoms to treatment (median } \\
\text { (IQR))—-days. } \\
\text { Clinical evolution ( } 24 \text { h before death) }\end{array}$ & $4.5(2.8-8.5)$ & $4(2-7)$ & 0.85 \\
\hline $\begin{array}{l}\text { Acute respiratory and radiological } \\
\text { worsening-no. }(\%)\end{array}$ & $18(72.0)$ & $280(94.3)$ & $<0.001$ \\
\hline Hemodynamic instability-no. (\%) & $7(29.2)$ & $108(37.1)$ & 0.44 \\
\hline New onset arrhythmias-no. (\%) & $0(0.0)$ & $8(2.8)$ & 1.00 \\
\hline Neurological deterioration-no. (\%) & $3(12.0)$ & $97(34.6)$ & 0.03 \\
\hline $\begin{array}{l}\text { Acute kidney injury-no. (\%) } \\
\text { Laboratory findings }\end{array}$ & $6(24.0)$ & $88(31.0)$ & 0.48 \\
\hline Creatinine at admission (mean (SD)) - $\mathrm{mg} / \mathrm{dL}$ & $2.3( \pm 2.6)$ & $1.6( \pm 1.4)$ & 0.04 \\
\hline $\begin{array}{l}\text { Maximum lymphocytopenia (median } \\
\text { (IQR))—cells } / \mathrm{mm} 3\end{array}$ & $500(300-675)$ & $500(300-700)$ & 0.50 \\
\hline Peak CRP (mean (SD))-mg/dL & $15.5( \pm 7.9)$ & $13.7( \pm 10.5)$ & 0.42 \\
\hline Peak TnI (mean (SD))—ng/mL & $0.26( \pm 0.5)$ & $2.6( \pm 27.3)$ & 0.73 \\
\hline Peak TnI > 0.05 ng $/ \mathrm{mL}$ - no. $(\%)$ & $9(56.3)$ & $96(51.6)$ & 0.72 \\
\hline Peak D-dimer (mean (SD))—ng/mL & $\begin{array}{c}12,207 \\
( \pm 28,511)\end{array}$ & $13,303( \pm 44,347)$ & 0.93 \\
\hline Peak ferritin (mean (SD))—ng/mL & $1.698( \pm 2030)$ & $1,359( \pm 1642)$ & 0.40 \\
\hline Peak triglycerides (mean (SD)) $-\mathrm{mg} / \mathrm{dL}$ & $139( \pm 148.6)$ & $327( \pm 726)$ & 0.67 \\
\hline IL-6 (median (IQR))—ng/mL & $\begin{array}{c}54.3 \\
(53.7-182.7)\end{array}$ & $103.2(47.7-253.7)$ & 1.00 \\
\hline INR ( $24 \mathrm{~h}$ before death) $\S$ (mean (SD)) & $2.3( \pm 4.5)$ & $1.3( \pm 0.7)$ & 0.004 \\
\hline $\begin{array}{l}\text { APTT (24 h before death) (mean (SD)) - } \mathrm{s} \\
\text { Radiological findings }\end{array}$ & $35.3( \pm 16.4)$ & $30.2( \pm 8.4)$ & 0.03 \\
\hline $\begin{array}{l}\text { Bilateral pneumonia } \\
\text { ECG abnormalities }\end{array}$ & $14(56.0)$ & $198(66.7)$ & 0.52 \\
\hline QTc (mean (SD)) -ms & $409( \pm 21)$ & $403( \pm 40)$ & 0.64 \\
\hline$\widehat{\mathrm{PR}}($ mean $(\mathrm{SD}))-\mathrm{ms}$ & $183( \pm 29)$ & $192( \pm 36)$ & 0.44 \\
\hline
\end{tabular}


Table 3. Cont.

\begin{tabular}{cccc}
\hline Univariable Analysis & $\begin{array}{c}\text { Unexpected } \\
\text { Deaths } \\
(\boldsymbol{n}=\mathbf{2 5})\end{array}$ & $\begin{array}{c}\text { Non-Unexpected } \\
\text { Deaths } \\
(\boldsymbol{n}=\mathbf{2 9 9 )}\end{array}$ & $\boldsymbol{p}$-Value \\
\hline $\begin{array}{c}\text { Treatments during the hospitalization } \\
\text { Hydroxychloroquine-no. (\%) }\end{array}$ & $17(68.0)$ & $198(66.0)$ & 0.87 \\
HCQ + Azithromycin-no. (\%) & $4(16.0)$ & $62(20.7)$ & 0.80 \\
HCQ + Lopinavir/ritonavir+ & $2(8.0)$ & $28(9.4)$ & 1.00 \\
Azithromycin-no. (\%) & $10(40)$ & $131(43.8)$ & 0.89 \\
Corticosteroids—no. (\%) & $1(4.0)$ & $14(4.7)$ & 1.00 \\
Tocilizumab-no. (\%) & $7(28.0)$ & $65(21.7)$ & 0.47 \\
Therapeutic anticoagulation-no. (\%) & $13(52.0)$ & $152(51.2)$ & 0.94 \\
Prophylactic anticoagulation-no. (\%) & $4(3-9)$ & $5(2-7.8)$ & 0.18 \\
Time from treatment to death (median & & & \\
(IQR))-days. & &
\end{tabular}

Values are $\mathrm{n}(\%)$, median (interquartile range) or mean (standard deviation) as indicated. * Previous admissions for heart failure. + Combination of immunosuppressive therapies (corticosteroids and other immunosuppressive drugs), transplanted patients and HIV+. ‡ At least one of: ACE-I, ARB, MRA, or sacubitril/valsartan. § Patients in treatment with AVK or DOAC during hospitalization were excluded. CRP: C-reactive protein, CVRF: cardiovascular risk factor, DVT: deep venous thrombosis, HCQ: hydroxychloroquine, MI: myocardial infarction, no: number, OAD: oral antidiabetic; PE: pulmonary embolism, RAAS: renin-angiotensin-aldosterone system, TIA: transient ischaemic attack, T2DM: type-2 diabetes mellitus, TnI: troponin I.

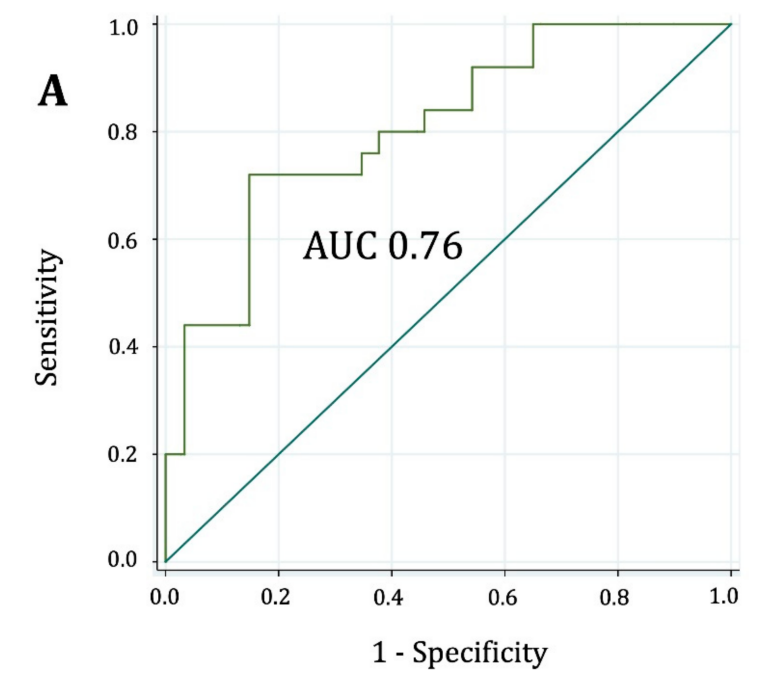

(A)

B

\begin{tabular}{|l|c|c|c|c|c|}
\hline \multicolumn{1}{|c|}{$\begin{array}{c}\text { Unexpected death prediction } \\
\text { model }\end{array}$} & OR & SE & \multicolumn{2}{c|}{$\boldsymbol{p}$-value } & \multicolumn{2}{c|}{ 95\% confidence interval } \\
\hline Male sex & 4.32 & 2.45 & 0.01 & 1.42 & 13.15 \\
\hline Diabetes mellitus insulin-dependent & 2.86 & 1.46 & 0.04 & 1.05 & 7.78 \\
\hline Chronic kidney disease & 2.34 & 1.08 & 0.06 & 0.95 & 5.80 \\
\hline Independent status & 2.71 & 1.43 & 0.06 & 0.96 & 7.65 \\
\hline
\end{tabular}

(B)

Figure 2. Unexpected death prediction model. (A) Represents the ROC curve with an area under the curve (AUC) of 0.75. Below, (B) Represents in a table the multivariable analysis with the corresponding values of odds ratio for each one of the four variables included in the model and their respective confidence intervals and $p$-values. OR: odds ratio, $\mathrm{SE}$ : standard error. 


\section{Discussion}

This hitherto largest series of consecutive non-ICU patients with COVID-19 who died in Spain illustrates that, as in the case of Italy [3], our patient population is older (median age 82.8 y) than the average age reported in Chinese patients [4,11,12]. Mortality increases with older age and this may partly explain differences in case-fatality rates among countries. The presence of pre-existing comorbidities was also particularly important in our series. Case fatality rate is much higher in patients with comorbidities [3,4,12-15]. In the current series, cardiovascular burden was also very prevalent (35.8\%), and main cardiovascular risk factors were frequently present, especially hypertension (78.4\%). A higher prevalence of hypertension in patients with poor outcome has also been reported [15], but when this association is adjusted for other risk factors, there is no strong evidence to indicate that hypertension is a predictor of mortality. Surprisingly, active smoking was very uncommon in our series $(n=15)$. In other COVID-19 series, it has also been lower than expected in a population with primary respiratory infection [16]. The percentage of obese patients $(18.5 \%)$ was much lower than in the series of the NY city area (41.7\%) [13].

One of the most relevant aspects is the high frailty of our patient's cohort. Almost $40 \%$ were partially or totally dependent in ADL and $15 \%$ were institutionalized. These data are, by extension, a reflection of what has happened in Madrid's region, as $30 \%$ of the deceased were institutionalized [17].

CURB-65 at admission, a clinical predictor score validated for predicting mortality in community-acquired pneumonia, was not accurate in our cohort $(60 \%$ had a score $\leq 2)$. The absence of tachypnea in many hypoxemic patients $\left(16.4 \%\right.$ and $40 \%$ with $\mathrm{O}_{2}$ saturation $<90 \%$ and $\leq 92 \%$ on room air, respectively), what could be called silent hypoxemia, and a stable hemodynamic situation at admission, might partially explain the low CURB-65 scores. Thus, compromised respiratory status at admission (the primary driver of pneumonia severity) was not the rule. To maintain hospital safety, imaging studies were not frequently performed. Thus, we do not have enough CT studies to completely depict these patient's pulmonary syndrome.

Corticosteroids and prophylactic heparin were more frequently used in the later, rather than in the early phase of the study, denoting that, as we became more knowledgeable about the characteristics of the disease (cytokine release storm and increased risk of venous thromboembolism), treatment changed.

In our series, $18 \%$ of patients could not benefit from ICU care due to lack of resources, and the same proportion of patients was pronated on the general ward. Similarly, a quarter of patients who died early in the Wuhan outbreak did not receive mechanical ventilation [18]. This clearly reflects the limitations of our healthcare systems, which were abruptly overwhelmed by a huge surge of patients needing mechanical ventilation. When resources are finite, differentiating whether the cause of the clinical situation is the viral infection or the patient's underlying condition is of paramount importance. Seventy-eight percent of our cohort were not ICU candidates due to their primary clinical situation. Regardless, ICU care for the elderly is not exactly a lifeline since, in some series with COVID-19, the mortality rate in patients over 65 years who required mechanical ventilation was $97 \%[12,13]$.

It is essential to assess the actual role of COVID-19 in patient's death. In 7.7\% of our cohort (Group C), the actual contribution of the virus to the patient's death was minimal, if any. In patients with terminal cancer, very advanced heart failure, multiorgan failure, etc., was the patient's underlying condition the cause of death, not the viral infection.

\section{Unexpected Death}

The actual cause of death is important, as the real course of the disease is not well known. Remarkably, 7.7\% of our cohort had an unexpected death. To the best of our knowledge, there are no recorded COVID-19 cases with such unexpected deaths.

All these patients had severe pneumonia and were more frequently male. Insulintreated diabetes, CKD and independency for ADL were independently associated with 
unexpected death. Several pathophysiological mechanisms (e.g., myocardial inflammation, coronary ischemia, arrhythmias, electrolyte imbalance, pulmonary embolism) can trigger these patients' death.

Cardiac arrest occurs in about 3\% of patients admitted with community-acquired pneumonia [19]. The most frequent risk factors of cardiac events in inpatients with pneumonia include older age, main cardiovascular risk factors, and greater severity of pneumonia at presentation [20]. All of them were present in our patient population. Cardiovascular risk factors, especially diabetes, were highly prevalent in patients with unexpected death. A recent meta-analysis showed a $75 \%$ increase in the relative risk of sudden death among diabetics compared to patients without diabetes. Pathogenesis involves macrovascular (coronary atherosclerosis), microvascular disease, and autonomic neuropathy [21].

Coronary artery disease, arrhythmias and sudden cardiac death represent the main causes of morbidity and mortality in patients with CKD. Pathogenesis involves the presence of traditional and non-traditional cardiovascular risk factors, including metabolic abnormalities [22]. Additionally, in our cohort, patients with CKD received prophylactic anticoagulation less frequently than those with normal renal function.

Though COVID-19 could be regarded as a disease that is confined to the lungs, cardiac complications, including myocardial infarction, myocarditis, conduction block and cardiac arrhythmias, have been reported $[11,16,23]$. Two patients with unexpected death had an ACS pointing to coronary artery disease (CAD) as the probable cause of death. However, increased troponin values did not significantly differ between unexpected and non-unexpected deaths. High levels of troponins have been associated with severe COVID19 pneumonia and death $[12,24]$. In most patients, the reported association could be more a consequence of the systemic inflammatory response than a sign of obstructive CAD. Antivirals used during the treatment of COVID-19 may increase the risk for arrhythmias and sudden death [25]. In this series, we did not find any differences between groups regarding treatment with these drugs or QTc prolongation.

Coagulopathy (increased D-dimer concentrations and prolongation of prothrombin and activated partial thromboplastin times) was frequently present, and, although not statistically significant in the multivariable analysis, it was more common in patients with unexpected death. Coagulopathy and pulmonary embolism (PE) are highly prevalent in COVID-19 [26-28]. Several studies focused on autopsies have confirmed that SARS-CoV-2 patients have a baseline hypercoagulable state and are at increased risk for pulmonary thrombotic microangiopathy as well as the development of deep vein thromboses and major pulmonary thromboembolism. The autopsy findings support evaluation and management for coagulopathy early in the course of disease and judicious use of prophylactic anticoagulants while hospitalized $[27,29]$. In a necropsy series of 11 patients in whom PE was not clinically suspected, thrombosis of small and mid-sized pulmonary arteries was found in all patients. Ten of them had received prophylactic anticoagulation [28].

Unexpected deaths were more frequent in the first study period along with less prophylactic anticoagulation. These data underpin PE as another potential cause of unexpected death.

We do not have an adequate explanation as to why independency for ADL was related to unexpected death. The prevalence of functional disability and its association with outcomes among patients with COVID-19 has not been studied. As in heart failure, COVID-19 patients with ADL difficulty might be more prone to die from underlying diseases and progressive respiratory failure than from cardiovascular causes. On the contrary, in independent subjects, a cardiovascular origin of death could be more likely [30]. Moreover, independent patients have more personal autonomy and require less hospital supervision, what would justify why three of them were found collapsed in the bathroom. There is little doubt that cardiac arrest in the bathroom has, in most cases, a cardiovascular cause [31].

Numerous uncertainties remain in our understanding of the management of COVID19 and on the causes of death, particularly sudden death. Improving understanding of 
the mechanism of death is critical. Meanwhile, we advocate for regular monitoring of independent diabetic males with CKD and severe COVID-19 pneumonia. Prophylactic anticoagulation is a must for all these patients. From a research perspective, the issues discussed highlight the need for autopsy reports to better understand this deadly disease.

\section{Conclusions}

This study provides a comprehensive overview of hospitalized non-ICU deceased patients with confirmed COVID-19 in the city of Madrid. The contribution of the virus infection to the patients' deaths has been assessed in detail. Some patients died unexpectedly and predictors of this type of death include male sex, insulin-treated diabetes, chronic kidney disease, and independence in activities of daily living. These results suggest that unexpected death in COVID-19 might be due to cardiovascular causes.

\section{Limitations}

This study has several limitations. Firstly, the study population only included non-ICU hospitalized deceased patients, so interpretation of our findings is limited to this patient population. Secondly, due to the retrospective study design, not all tests were recruited in all patients. Therefore, their role, especially the $48 \mathrm{~h}$ ECG after admission, might be underestimated in predicting unexpected death. Moreover, as patients were admitted to general medical wards, continuous monitoring was not available.

Supplementary Materials: Supplementary materials can be found at https:/ / www.mdpi.com/20 77-0383/10/4/825/s1. Supplementary Figure S1. Observed versus predicted unexpected death. Observed unexpected deaths are represented by blue bars and predicted unexpected deaths by yellow bars, divided in quintiles. Supplementary Table S1. Baseline medications. Supplementary Table S2. Radiological findings.

Author Contributions: Conceptualization: methodology, investigation, data curation, formal analysis, writing—original draft preparation, review and editing: C.N.P.-G., I.V., M.M.-B., D.E.-V. Investigation and data curation: C.O., J.C.G.-P., R.I., N.R.-L., J.L.G.-K., M.F.-E., A.J., E.M.-G., J.F.-U., M.F.-S., P.P.-A., T.R.-D., Z.G.-Á., J.P.-E., E.J., G.V., E.G., E.O., I.B., A.C. Formal analysis, review, editing, and supervision: Á.N., Á.M., N.L.-V., E.A., C.M. All authors have read and agreed to the published version of the manuscript.

Funding: This research received no external funding.

Institutional Review Board Statement: The study was conducted according to the guidelines of the Declaration of Helsinki, and approved by the Institutional Ethics Committee of Hospital Clínico San Carlos (protocol code 20/342-E_COVID, 17 April 2020).

Informed Consent Statement: Not applicable, due to the aim and the characteristics of the study design.

Data Availability Statement: The data presented in this study are available on request from the corresponding author.

Acknowledgments: We would first like to acknowledge our medical colleagues and nurses for their excellent work during this sad and hard episode of our professional life. Working all together has been the best multidisciplinary experience. We are also grateful to the Departments of Cardiology and Internal Medicine from the Hospital Clínico San Carlos for their support.

Conflicts of Interest: The authors declare no conflict of interest.

$\begin{array}{ll}\text { Abbreviations } & \\ \text { ACE-i } & \text { angiotensin-converting enzyme inhibitor } \\ \text { ACS } & \text { acute coronary syndrome } \\ \text { ADL } & \text { activities of daily living } \\ \text { APTT } & \text { activated partial thromboplastin time } \\ \text { ARB } & \text { angiotensin II receptor blocker }\end{array}$




\begin{tabular}{|c|c|}
\hline AUC & area under the curve \\
\hline AVK & antivitamin $\mathrm{K}$ \\
\hline $\mathrm{BP}$ & blood pressure \\
\hline BUN & blood urea nitrogen \\
\hline CAD & coronary artery disease \\
\hline CI & confidence interval \\
\hline CKD & chronic kidney disease \\
\hline COPD & chronic obstructive pulmonary disease \\
\hline CPD & chronic pulmonary disease \\
\hline CRP & C-reactive protein \\
\hline CRT & cardiac resynchronization therapy \\
\hline CTA & computed tomography angiography \\
\hline CVRF & cardiovascular risk factor \\
\hline CURB-65 & confusion, BUN, respiratory rate, systolic blood pressure, age $\geq 65$ \\
\hline DOAC & direct oral anticoagulant \\
\hline DVT & deep venous thrombosis \\
\hline ECG & electrocardiogram \\
\hline ED & emergency department \\
\hline HCQ & hydroxychloroquine \\
\hline $\mathrm{ICD}$ & implantable cardiac device \\
\hline ICU & intensive care unit \\
\hline INR & international normalized ratio \\
\hline IQR & interquartile range \\
\hline LVEF & left ventricular ejection fraction \\
\hline MI & myocardial infarction \\
\hline MRA & mineralocorticoid receptor antagonist \\
\hline no & number \\
\hline OAD & oral antidiabetic \\
\hline OSAS & obstructive sleep apnea syndrome \\
\hline OR & odds ratio \\
\hline PE & pulmonary embolism \\
\hline RAAS & renin-angiotensin-aldosterone system \\
\hline ROC & receiver operating characteristic \\
\hline RT-PCR & reverse transcriptase-polymerase chain reaction \\
\hline SARS-CoV-2 & severe acute respiratory syndrome coronavirus 2 \\
\hline SD & standard deviation \\
\hline SE & standard error \\
\hline TIA & transient ischaemic attack \\
\hline TnI & troponin I \\
\hline T2DM & type-2 diabetes mellitus \\
\hline SARS-CoV-2 & severe acute respiratory syndrome-coronavirus 2 \\
\hline
\end{tabular}

\section{References}

1. Johns Hopkins University Coronavirus Resource Center. COVID-19 Dashboard by the Center for Systems Science and Engineering (CSSE) at Johns Hopkins University (JHU). Available online: https:// coronavirus.jhu.edu/map.html (accessed on 7 February 2021).

2. España. Ministerio de Sanidad. Consumo y Bienestar Social, 'Situación Actual'. Available online: https://www.mscbs.gob.es/ profesionales/saludPublica/ccayes/alertasActual/nCov/situacionActual.htm (accessed on 7 February 2021).

3. Onder, G.; Rezza, G.; Brusaferro, S. Case-Fatality Rate and Characteristics of Patients Dying in Relation to COVID-19 in Italy. JAMA 2020, 323, 1775-1776. [CrossRef]

4. Ruan, Q.; Yang, K.; Wang, W.; Jiang, L.; Song, J. Clinical predictors of mortality due to COVID-19 based on an analysis of data of 150 patients from Wuhan, China. Intensive Care Med. 2020, 46, 846-848. [CrossRef]

5. British Thoracic Society Standards of Care Committee. BTS Guidelines for the management of community acquired pneumonia in adults. Thorax 2001, 56, IV1-IV64. [CrossRef]

6. Tuijl, J.P.; Scholte, E.M.; de Craen, A.J.M.; van der Mast, R.C. Screening for cognitive impairment in older general hospital patients: Comparison of the Six-Item Cognitive Impairment Test with the Mini-Mental State Examination. Int. J. Geriatr. Psychiatry 2012, 27, 755-762. [CrossRef] [PubMed] 
7. Singer, M.; Deutschman, C.S.; Seymour, C.W.; Shankar-Hari, M.; Annane, D.; Bauer, M.; Bellomo, R.; Bernard, G.R.; Chiche, J.-D.; Coopersmith, C.M.; et al. The Third International Consensus Definitions for Sepsis and Septic Shock (Sepsis-3). JAMA 2016, 315, 801-810. [CrossRef]

8. Williams, B.; Mancia, G.; Spiering, W.; Rosei, E.A.; Azizi, M.; Burnier, M.; Clement, D.L.; Coca, A.; de Simone, G.; Dominiczak, A.F.; et al. 2018 ESC/ESH Guidelines for the management of arterial hypertension. Eur. Heart J. 2018, 39, 3021-3104. [CrossRef] [PubMed]

9. Force, A.D.; Ranieri, V.M.; Rubenfeld, G.D.; Thompson, B.; Ferguson, N.; Caldwell, E. Acute respiratory distress syndrome: The Berlin Definition. JAMA 2012, 307, 2526-2533. [CrossRef]

10. Winkel, B.G.; Holst, A.G.; Theilade, J.; Kristensen, I.B.; Thomsen, J.L.; Ottesen, G.L.; Bundgaard, H.; Svendsen, J.H.; Haunsø, S.; Tfelt-Hansen, J. Nationwide study of sudden cardiac death in persons aged 1-35 years. Eur. Hear. J. 2010, 32, 983-990. [CrossRef] [PubMed]

11. Wang, D.; Hu, B.; Hu, C.; Zhu, F.; Liu, X.; Zhang, J.; Wang, B.; Xiang, H.; Cheng, Z.; Xiong, Y.; et al. Clinical Characteristics of 138 Hospitalized Patients With 2019 Novel Coronavirus-Infected Pneumonia in Wuhan, China. JAMA 2020, 323, 1061-1069. [CrossRef]

12. Zhou, F.; Yu, T.; Du, R.; Fan, G.; Liu, Y.; Liu, Z.; Xiang, J.; Wang, Y.; Song, B.; Gu, X.; et al. Clinical course and risk factors for mortality of adult inpatients with COVID-19 in Wuhan, China: A retrospective cohort study. Lancet 2020, 395, 1054-1062. [CrossRef]

13. Richardson, S.; Hirsch, J.S.; Narasimhan, M.; Crawford, J.M.; McGinn, T.; Davidson, K.W.; The Northwell COVID-19 Research Consortium. Presenting Characteristics, Comorbidities, and Outcomes among 5700 Patients Hospitalized With COVID-19 in the New York City Area. JAMA 2020, 323, 2052-2059. [CrossRef]

14. Grasselli, G.; Zangrillo, A.; Zanella, A.; Antonelli, M.; Cabrini, L.; Castelli, A.; Cereda, D.; Coluccello, A.; Foti, G.; Fumagalli, R.; et al. Baseline characteristics and outcomes of 1591 patients infected with SARS-CoV-2 admitted to ICUs of the Lombardy region, Italy. JAMA 2020, 323, 1574-1581. [CrossRef] [PubMed]

15. Epidemiology Working Group for NCIP Epidemic Response. The epidemiological characteristics of an outbreak of 2019 novel coronavirus diseases (COVID-19) in China. China J. Epidemiol. 2020, 41, 145-151. [CrossRef]

16. Guzik, T.J.; Mohiddin, S.A.; DiMarco, A.; Patel, V.; Savvatis, K.; Marelli-Berg, F.M.; Madhur, M.S.; Tomaszewski, M.; Maffia, P.; D'Acquisto, F.; et al. COVID-19 and the cardiovascular system: Implications for risk assessment, diagnosis, and treatment options. Cardiovasc. Res. 2020, 116, 1666-1687. [CrossRef]

17. Dirección General de Salud Pública, Servicio Madrileño de Salud y Hospitales Privados. Available online: http:/ / comunidad. madrid/servicios/salud/2019-nuevo-coronavirus (accessed on 7 February 2021).

18. Xie, J.; Tong, Z.; Guan, X.; Du, B.; Qiu, H.; Slutsky, A.S. Critical care crisis and some recommendations during the COVID-19 epidemic in China. Intensiv. Care Med. 2020, 46, 837-840. [CrossRef]

19. Marrie, T.J.; Shariatzadeh, M.R. Community-Acquired Pneumonia Requiring Admission to an Intensive Care Unit. Medicine 2007, 86, 103-111. [CrossRef]

20. Corrales-Medina, V.F.; Musher, D.M.; Wells, G.A.; Chirinos, J.A.; Chen, L.; Fine, M.J. Cardiac Complications in Patients with Community-Acquired Pneumonia. Circulation 2012, 125, 773-781. [CrossRef]

21. Aune, D.; Schlesinger, S.; Norat, T.; Riboli, E. Diabetes mellitus and the risk of sudden cardiac death: A systematic review and meta-analysis of prospective studies. Nutr. Metab. Cardiovasc. Dis. 2018, 28, 543-556. [CrossRef]

22. Di Lullo, L.; House, A.; Gorini, A.; Santoboni, A.; Russo, D.; Ronco, C. Chronic kidney disease and cardiovascular complications. Heart Fail Rev. 2015, 20, 259-272. [CrossRef] [PubMed]

23. Zheng, Y.-Y.; Ma, Y.-T.; Zhang, J.-Y.; Xie, X. COVID-19 and the cardiovascular system. Nat. Rev. Cardiol. 2020, 17, 259-260. [CrossRef]

24. Lippi, G.; Lavie, C.J.; Sanchis-Gomar, F. Cardiac troponin I in patients with coronavirus disease 2019 (COVID-19): Evidence from a meta-analysis. Prog. Cardiovasc. Dis. 2020, 63, 390-391. [CrossRef]

25. Ray, W.A.; Murray, K.T.; Hall, K.; Arbogast, P.G.; Stein, C.M. Azithromycin and the Risk of Cardiovascular Death. N. Engl. J. Med. 2012, 366, 1881-1890. [CrossRef] [PubMed]

26. Bowles, L.; Platton, S.; Yartey, N.; Dave, M.; Lee, K.; Hart, D.P.; Macdonald, V.; Green, L.; Sivapalaratnam, S.; Pasi, K.J.; et al. Lupus Anticoagulant and Abnormal Coagulation Tests in Patients with Covid-19. N. Engl. J. Med. 2020, 383, 288-290. [CrossRef]

27. Buja, L.M.; Wolf, D.A.; Zhao, B.; Akkanti, B.; McDonald, M.; Lelenwa, L.; Reilly, N.; Ottaviani, G.; Elghetany, M.T.; Trujillo, D.O.; et al. The emerging spectrum of cardiopulmonary pathology of the coronavirus disease 2019 (COVID-19): Report of 3 autopsies from Houston, Texas, and review of autopsy findings from other United States cities. Cardiovasc. Pathol. 2020, $48,107233$. [CrossRef]

28. Lax, S.F.; Skok, K.; Zechner, P.; Kessler, H.H.; Kaufmann, N.; Koelblinger, C.; Vander, K.; Bargfrieder, U.; Trauner, M. Pulmonary Arterial Thrombosis in COVID-19 With Fatal Outcome. Ann. Intern. Med. 2020, 173, 350-361. [CrossRef]

29. Thachil, J. The versatile heparin in COVID-19. J. Thromb. Haemost. 2020, 18, 1020-1022. [CrossRef] [PubMed]

30. Dunlay, S.M.; Manemann, S.M.; Chamberlain, A.M.; Cheville, A.L.; Jiang, R.; Weston, S.A.; Roger, V.L. Activities of Daily Living and Outcomes in Heart Failure. Circ. Hear. Fail. 2015, 8, 261-267. [CrossRef]

31. Inamasu, J.; Miyatake, S. Cardiac arrest in the toilet: Clinical characteristics and resuscitation profiles. Environ. Health Prev. Med. 2013, 18, 130-135. [CrossRef] [PubMed] 